EPJ Web of Conferences 73, 06005 (2014)

DOI: $10.1051 /$ epjconf/20147306005

(C) Owned by the authors, published by EDP Sciences, 2014

\title{
The multiple-scattering series in few-nucleon systems
}

\author{
V. Baru ${ }^{1,2, a}$, E. Epelbaum ${ }^{1}$, C. Hanhart ${ }^{3,4}$, M. Hoferichter ${ }^{5}$, A.E. Kudryavtsev² and D.R. Phillips ${ }^{6}$ \\ ${ }^{1}$ Institut für Theoretische Physik II, Ruhr-Universität Bochum, 44780 Bochum, Germany \\ ${ }^{2}$ Institute for Theoretical and Experimental Physics, B. Cheremushkinskaya 25, 117218 Moscow, \\ Russia \\ ${ }^{3}$ Institut für Kernphysik and Jülich Center for Hadron Physics, Forschungszentrum Jülich, \\ 52425 Jülich, Germany \\ ${ }^{4}$ Institute for Advanced Simulation, Forschungszentrum Jülich, 52425 Jülich, Germany \\ ${ }^{5}$ Albert Einstein Center for Fundamental Physics, Institute for Theoretical Physics, University of Bern, \\ Sidlerstrasse 5, 3012 Bern, Switzerland \\ ${ }^{6}$ Institute of Nuclear and Particle Physics and Department of Physics and Astronomy, Ohio University, \\ Athens, OH 45701, USA
}

\begin{abstract}
We discuss under which circumstances the resummation of the multiplescattering series is justified from an EFT point of view. The application to $\pi d$ and $\bar{K} d$ scattering is briefly discussed.
\end{abstract}

\section{Introduction}

The multiple-scattering series (MSS) is a particular class of diagrams where a meson (e.g. a pion) scatters many times between a pair of other particles such as nucleons, see Fig. 1 for illustration. This class of diagrams is especially relevant for an accurate description of meson-nucleus scattering, which is an important source of information about meson-nucleon (MN) scattering lengths [1-3]. With the recent advances of effective field theories, in particular chiral perturbation theory (ChPT), an accurate extraction of the MN scattering lengths with a controlled uncertainty became possible. In particular, the $\pi N$ scattering lengths were extracted from a recent ChPT analysis of pionic hydrogen and deuterium atomic data with an unprecedented accuracy [1,2]. Such an analysis calls for rigorous control over higher-order ChPT corrections to pion-nucleus scattering with higher-order terms of the MSS representing the most prominent ones.

In this paper we follow Ref. [4] to demonstrate that diagrams of the MSS topology are a factor of $\pi^{2}$ larger compared to the standard ChPT counting predictions. This finding raise a question of the theoretical uncertainty estimate. The consequences of this enhancement for meson-deuteron scattering with a natural MN scattering length are discussed. In particular, we compare the results of the resummed MSS with a perturbative approach for $\pi d$ scattering and discuss some insights into $\bar{K} d$ scattering.

\footnotetext{
ae-mail: vadimb@tp2.rub.de
}

This is an Open Access article distributed under the terms of the Creative Commons Attribution License 4.0, which permits unrestricted use, distribution, and reproduction in any medium, provided the original work is properly cited. 


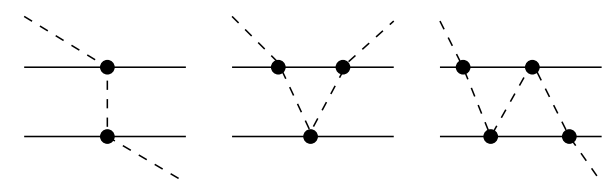

Figure 1. Second, third, and fourth term in the MSS. Solid lines denote nucleons, dashed mesons.

\section{MSS in perturbation theory}

Already in 1953 Brückner pointed out that the MSS should provide a large contribution to pion-deuteron scattering [5]. Weinberg came to the same conclusion [7] within the ChPT classification of diagrams based on a systematic, model independent expansion of the amplitudes in terms of momenta and pion masses measured in units of $\Lambda \sim 4 \pi F_{\pi} \sim 1 \mathrm{GeV}$. In particular, the first diagram of Fig. 1, the so-called double-scattering term, appears already at leading order (LO) in the chiral expansion of the 3-body $(\pi N N)$ operators [7], while the next diagram, the triple-scattering term, is suppressed by two orders in the expansion $\left(\mathrm{N}^{2} \mathrm{LO}\right)$.

Indeed, as long as nucleons are considered static and the incoming (outgoing) meson is at rest, the explicit evaluation of the double- and triple-scattering ${ }^{1}$ terms yields $[4,8]$

$$
\begin{aligned}
& A^{(2)}(\mathbf{Q})=\frac{a^{2}}{\mathbf{Q}^{2}}, \\
& A^{(3)}(\mathbf{Q})=-4 \pi a^{3} \int \frac{d^{3} l}{(2 \pi)^{3}} \frac{1}{\mathbf{l}^{2}(\mathbf{l}-\mathbf{Q})^{2}}=-\frac{a^{3}}{2 \pi|\mathbf{Q}|} J_{0},
\end{aligned}
$$

where $\mathbf{Q}=\mathbf{p}^{\prime}-\mathbf{p}$ denotes the three-momentum transfer between the incoming and outgoing nucleons, and $a \simeq M_{\pi} /\left(8 \pi F_{\pi}^{2}\right)$ is used as a measure of natural MN scattering lengths. Assuming for the dimensionless integral $J_{0}$ to be of order 1 , one finds the parametric suppression of the triple-scattering term predicted by Weinberg's power counting

$$
\frac{A^{(3)}}{A^{(2)}}=-\frac{a Q}{2 \pi} J_{0} \sim\left(\frac{M_{\pi}}{4 \pi F_{\pi}}\right)^{2} J_{0}
$$

However, an explicit evaluation of $J_{0}$,

$$
J_{0}=\int_{0}^{\infty} \frac{d x}{x} \log \left(\frac{x+1}{x-1}\right)^{2}=\pi^{2},
$$

leads to the numerical enhancement of $A^{(3)}$. To account for this effect the triple-scattering term was promoted to next-to-leading (NLO) order in Ref. [1,2]. The origin of the enhancement was identified in Ref. [8] with the specific topology of the triple-scattering term consisting of two consecutive pion exchanges with Coulombic-type (no pion mass scale) pion propagators. This leads to concerns regarding the enhancement of the quadruple-scattering term, which up to higher-order terms reads

$$
A^{(4)}(\mathbf{Q})=(4 \pi)^{2} a^{4} \int \frac{d^{3} l_{1}}{(2 \pi)^{3}} \frac{d^{3} l_{2}}{(2 \pi)^{3}} \frac{1}{\mathbf{l}_{1}^{2}\left(\mathbf{l}_{1}-\mathbf{l}_{2}\right)^{2}\left(\mathbf{l}_{2}-\mathbf{Q}\right)^{2}}=-a^{4} \log \frac{Q}{\mu}+\frac{f_{0}(\mu)}{32 \pi^{2}} .
$$

The divergent integral in Eq. (5) was first regularized and then renormalized by adding the regulatordependent contact term $f_{0}(\mu)$. The comparison of Eqs. (1) and (5) reveals also the $\pi^{2}$-enhancement of

\footnotetext{
${ }^{1}$ In the evaluation of the triple-scattering term only the relevant for our work (enhanced) contribution is kept. The full result is given in Ref. [8], where it is also shown that the residual terms are of natural size.
} 
the quadruple-scattering term

$$
\frac{A^{(4)}}{A^{(2)}}=a^{2} Q^{2} \sim \pi^{2}\left(\frac{M_{\pi}}{4 \pi F_{\pi}}\right)^{4} .
$$

This kind of enhancement takes place also at higher MSS orders, as discussed in Ref. [4]. The observed numerical enhancement of the MSS diagrams is potentially troublesome for the theoretical uncertainty estimate and predictive power of the theory, since it may suggest the existence of larger-than-expected contact operators.

\section{MSS resummation}

A solution to this problem was proposed in Ref. [4], where it was shown that the UV divergences of the individual MSS terms cancel once the whole series is resummed. Hence, no enhanced short-range operators are expected to contribute in the resummed MSS.

The multiple scattering amplitude for a meson scattering from the static $N N$ pair can be written as

$$
A(\mathbf{Q})=t_{\pi N}(0) \frac{\pi}{2} \delta^{(3)}(\mathbf{Q})+\int \frac{d^{3} p^{\prime \prime}}{(2 \pi)^{3}} t_{\pi N}\left(\mathbf{p}-\mathbf{p}^{\prime \prime}\right) \frac{1}{\left(\mathbf{p}-\mathbf{p}^{\prime \prime}\right)^{2}} A\left(\mathbf{p}^{\prime}-\mathbf{p}^{\prime \prime}\right),
$$

where the elementary MN amplitude reads $t_{\pi N}(\mathbf{p})=-4 \pi a \hat{g}\left(\frac{|\mathbf{p}|}{\Lambda_{\pi N}}\right)$, and $\hat{g}(x)$ takes into account a nonzero range of $\mathrm{MN}$ interaction $(\hat{g}(x) \rightarrow 1$ when $x \rightarrow 0)$. From an EFT point of view $\Lambda_{\pi N}$ plays the role of a regulator, and Eq. (7) is valid when $Q<\Lambda_{\pi N} \simeq \Lambda$. Taking the Fourier transform of Eq. (7), one finds the solution for the resummed amplitude in coordinate space

$$
A(r)=-\frac{a r}{4 \pi(r+a g(r))} .
$$

From this one can draw the following conclusions

- The perturbative treatment of Eq. (7) is justified as long as the MN scattering length is natural

$$
a \lesssim \Lambda^{-1} \simeq\left(4 \pi F_{\pi}\right)^{-1} \Longrightarrow \frac{a}{r} \sim \frac{Q}{\Lambda} \ll 1
$$

- The expansion of Eq. (8) in $a / r$ with the subsequent Fourier transform to momentum space reproduces the individual MSS terms discussed in Sect. 2.

- The resummed MSS (Eq. (8)) is much less regulator dependent than the individual contributions.

- For $\pi d$ scattering taking the limit $\Lambda_{\pi N} \rightarrow \infty$ in Eq. (8) results in the correction to the scattering length

$$
\langle\Delta A\rangle \equiv\left\langle A_{\Lambda_{\pi N}}(r)-A_{\Lambda_{\pi N} \rightarrow \infty}(r)\right\rangle \quad \leq 3 \% a_{\pi d}
$$

This is fully in line with the estimate of the (natural) contact term at $\mathrm{N}^{2} \mathrm{LO}$ in chiral counting $[1,2,4]$.

\section{A problem with the pole}

The resummed MSS result exhibits an unphysical pole for attractive MN interaction $(a<0)$ since the denominator of Eq. (8) may go to zero in this case, ${ }^{2}$ see Fig. 2. This can be naively interpreted as a

\footnotetext{
${ }^{2}$ A similar effect is discussed in Ref. [9] as the result of the solution of the momentum-space integral equation.
} 
Figure 2. Position of the pole at $r=$ $-a g(r)$ for $a<0$.

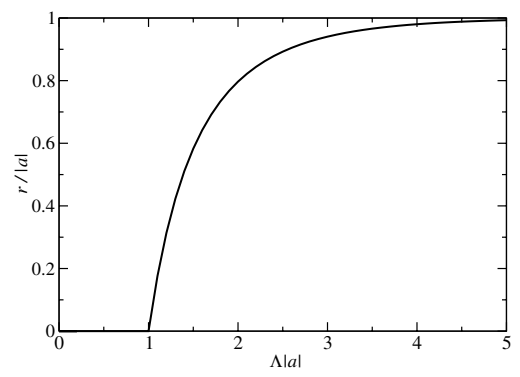

failure of the resummed results. However, for a natural scattering length the pole occurs very close to the origin for $r \sim a<\Lambda^{-1}$ or $Q>\Lambda$, i.e. it is already beyond the region of applicability of the EFT. Thus we believe that the MSS delivers reliable results as long as $a$ is natural. On the contrary, for an unnaturally large and negative scattering length $(a \Lambda>1)$ the pole indeed appears in the physical region $r \sim Q^{-1} \sim a>\Lambda^{-1}$, but as long as $Q^{-1} \sim a$ a shallow MN state can be formed. Therefore, the 2-body input to the integral equation (7) should be modified to include at least the unitarity corrections

$$
t_{\pi N}(\mathbf{p})=-\frac{4 \pi}{1 / a-i p} \hat{g}\left(\frac{|\mathbf{p}|}{\Lambda_{\pi N}}\right) .
$$

In addition, nucleon recoil effects should also be considered [10]. We expect that keeping these effects would shift the pole towards the origin, i.e. outside the physical region.

\section{Some applications}

Due to the natural size of the $\pi N$ scattering length both perturbative and non-perturbative treatments are applicable to $\pi d$ scattering: in the perturbative case the numerical enhancement of the short-range operator turns out to be not sufficient to overcome the strong parametric suppression of the quadruplescattering term, see Eq. (6), while the non-perturbative scheme is applicable since the pole does not affect the results. Given the larger magnitude of the kaon-nucleon scattering lengths $(\sim 1 \mathrm{fm})$, one may wonder if the resummed approach is applicable to kaonic deuterium [11, 12]. We believe that it does apply for the following reasons: first, in the case of isovector dominated and/or absorptive interactionsboth true for $\bar{K} N$ interactions-no pole appears on the real axis. Second, the natural hard scale in the EFT for the $\bar{K} d$ system is $\Lambda \sim 2 M_{\pi}$ [13], which means that $\Lambda a \sim 1$ and the pole should lie not far from the origin, see Fig. 2.

This work is supported by the EU via the FP7 HadronPhysics3 and ERC-2010-StG 259218 NuclearEFT projects, by the DFG through funds provided to the Sino-German CRC 110.

\section{References}

[1] V. Baru, C. Hanhart, M. Hoferichter, B. Kubis, A. Nogga and D.R. Phillips, Nucl. Phys. A 872, $69(2011)$

[2] V. Baru, C. Hanhart, M. Hoferichter, B. Kubis, A. Nogga and D.R. Phillips, Phys. Lett. B 694, $473(2011)$

[3] J. Gasser, V.E. Lyubovitskij and A. Rusetsky, Phys. Rept. 456, 167 (2008)

[4] V. Baru, E. Epelbaum, C. Hanhart, M. Hoferichter, A.E. Kudryavtsev and D.R. Phillips, Eur. Phys. J. A 48, 69 (2012) 
[5] K.A. Brueckner, Phys. Rev. 89, 834 (1953)

[6] K.A. Brueckner, Phys. Rev. 90, 715 (1953)

[7] S. Weinberg, Phys. Lett. B 295, 114 (1992)

[8] S. Liebig, V. Baru, F. Ballout, C. Hanhart and A. Nogga, Eur. Phys. J. A 47, 69 (2011)

[9] A.E. Kudryavtsev, A.I. Romanov and V.A. Gani, Phys. Atom. Nucl. 76, 919 (2013)

[10] V. Baru, C. Hanhart, A.E. Kudryavtsev and U.-G. Meißner, Phys. Lett. B 589, 118 (2004)

[11] S.S. Kamalov, E. Oset and A. Ramos, Nucl. Phys. A 690, 494 (2001)

[12] U.-G. Meißner, U. Raha and A. Rusetsky, Eur. Phys. J. C 47, 473 (2006)

[13] V. Baru, E. Epelbaum and A. Rusetsky, Eur. Phys. J. A 42, 111 (2009) 